\title{
Entrepreneurship
}

Jul a Dez $2021-$ v.5 - n.2

ISSN: 2595-4318

This article is also available online at: www.sustenere.co

\section{Significações da implantação de ações ecoinovadoras em empresas do ramo madeireiro da região Sul do Estado do Paraná}

A pesquisa teve por objetivo identificar as significações causadas pela implementação de ações ecoinovadoras dentro do processo produtivo de empresas do segmento madeireiro da Região Centro Sul do Paraná, sob os aspectos ambientais e econômicos. Apurou-se o conhecimento sobre ecoinovação, bem como a efetividade das ações adotadas pelas empresas pesquisadas. Quanto à metodologia, utilizou se a abordagem qualitativa e de análise exploratória. Os resultados apontam para uma maior conscientização e cuidado do meio ambiente, por parte dos gestores, quando e na hipótese de alterações nos processos produtivos. Economicamente, proteger o meio ambiente significa ir além da valorização do produto no mercado externo, mas promover o desenvolvimento sustentável e com qualidade de vida do entorno.

Palavras-chave: Ecoinovação; Sustentabilidade; Desenvolvimento Sustentável; Gestão Ambiental.

\section{Significations of eco-innovative actions implementation in the wood industry in the South of Paraná}

The research aimed to identify the significations caused by the implementation of eco-innovative actions within the production process of wood companies in the South area of Paraná, regarding the environmental and economic aspects. The research investigated the surveyed companies; knowledge of eco-innovation, as well as, the effectiveness of the actions implemented by them. Regarding the methodology, it used qualitative and exploratory analysis. The results pointed to greater awareness and environmental care by the managers when and in the hypothesis of changes in the production processes. Economically, protecting the environment means going beyond the product value in the external market, but promoting sustainable development and granting life quality to the surrounded areas.

Keywords: Eco-innovation; Sustainability; Sustainable Development; Environmental Management.

Topic: Planejamento, Estratégia e Competitividade

Reviewed anonymously in the process of blind peer.
Received: 11/07/2021

Approved: $25 / 09 / 2021$
Amanda Ortiz Pordeus

Universidade Estadual do Centro-Oeste, Brasil

http://lattes.cnpq.br/4838970111838430

http://orcid.org/0000-0003-4458-8486

amanda_pordeus@hotmail.com

Telma Regina Stroparo (iD

Universidade Estadual do Centro-Oeste, Brasil

http://lattes.cnpq.br/5911059392094700

http://orcid.org/0000-0001-8446-992X

telma@unicentro.br
Referencing this:

PORDEUS, A. O.; STROPARO, T. R.. Significações da implantação de ações ecoinovadoras em empresas do ramo madeireiro da região Sul do Estado do Paraná. Entrepreneurship, v.5, n.2, p.56-62, 2021. DOI: http://doi.org/10.6008/CBPC2595-4318.2021.002.0005 


\section{INTRODUÇÃO}

Ecoinovação pode ser considerada como a introdução de produtos, processos produtivos, métodos de gestão ou negócios, novos ou significativamente melhorados, para a organização e que traz benefícios econômicos, sociais e ambientais, comparados com alternativas pertinentes (FARIAS et al., 2012).

Discute-se os meios de produção e busca-se alternativas de desenvolvimento quer econômico, quer territorial, que preserve a natureza, não polua e não degrade áreas do meio ambiente. No entanto, entendese que o debate é mais amplo e obviamente inclui o âmbito das empresas e a sociedade de um modo geral, no sentido de que estas devem buscar o desenvolvimento com melhores condições de trabalho e que não causem males aos empregados como por exemplo, a instalação de filtros de fumaça, diminuição de poeiras e resíduos tóxicos, etc.

O grande desafio das organizações é desenvolver-se, inovar e incorporar a sustentabilidade em seus processos e estratégias de negócio, a fim de obter melhores vantagens competitivas (KNEIPP et al., 2011).

Defende-se aqui que os processos produtivos sejam revistos e, na medida do possível, adequados com ações ecoinovadoras, com padrões de produção e consumo que possam reduzir os impactos adversos ao meio ambiente e sociedade pois empresas assim consideradas são capazes de vincular suas principais estratégias tecnológicas, de inovação e de negócios com consequente vantagem competitiva (LAWSON et al., 2001; OECD, 2009).

Tratando especificamente sobre o segmento madeireiro verifica-se a crescente preocupação com a exploração ambientalmente adequada, com utilização de métodos menos invasivos e com utilização de tecnologia capaz de reduzir os impactos de degradação bem como ações de recomposição e a consequente implementação de ações ecoinovadoras. Verifica-se, no entanto, conforme pesquisa de Maçaneiro et al. (2015) que há um longo caminho para estudos e sistematização das estatísticas relacionadas ao tema.

Diversos estudos científicos tratam do assunto, dentre os quais citam-se Rennings (1998); Sharma et al. (1999); Sales-Campos et al. (2000); Spaargaren (2003); Spathelf et al. (2004); Arundel et al. (2009); Barbieri et al. (2010); Barbieri (2011); Maçaneiro et al. (2015), mas mesmo assim o estudo reveste-se de importância por tratar especificamente do setor madeireiro, segmento economicamente importante para o Estado do Paraná, e por investigar etnograficamente percepções de gestores e empregados sobre ações implementadas no âmbito de suas empresas, discutir efetividade e entendimento (significações) que adveio.

Dessa forma, a pesquisa tem por objetivo identificar as significações causadas pela implementação de ações ecoinovadoras dentro do processo produtivo de empresas do segmento madeireiro da Região Centro Sul do Paraná, sob os aspectos ambientais e econômicos e está estruturada em quatro seções incluindo esta introdução. A seguir apresenta-se a fundamentação teórica sobre ecoinovações, sustentabilidade, desenvolvimento sustentável e segmento madeireiro. Após, na seção 3, descreve-se o método adotado e os resultados são apresentados e discutidos e, por fim, na seção 4, tem-se as considerações finais. 


\section{METODOLOGIA}

Nesta seção são apresentados os conceitos teóricos que fundamentam a pesquisa e versam sobre Ecoinovações, Sustentabilidade, Desenvolvimento Sustentável e segmento madeireiro.

\section{Ecoinovação, Sustentabilidade e Desenvolvimento Sustentável}

O conceito de ecoinovação é relativamente novo e consiste no desenvolvimento e aplicação de um modelo de negócios forjado por uma estratégia que incorpora a sustentabilidade em suas operações do negócio. Engloba um conjunto coordenado de modificações ou novas soluções para os produtos (bens/serviços), processos, abordagem de mercado e estrutura organizacional que leve a um maior desempenho e competitividade da empresa (UNEP, 2014).

A definição de inovação que incorpora a dimensão ambiental perpassa os temas de diagnóstico, prevenção da poluição, redução do passivo ambiental e impacto ambiental, sendo que a maior parte dos conceitos incorpora a redução dos efeitos ambientais (SCHIEDERIG et al., 2012; PINSKY et al., 2015; KOELLER et al., 2020).

Segundo Maçaneiro et al. (2010) A ecoinovação é uma inovação que resulta em uma redução do impacto ambiental, sendo esses impactos intencionais ou não, sendo assim, uma maneira de inovações sustentáveis. Quanto à utilização do termo ecoinovação tem-se a pesquisa de Fussler e James no livro Driving eco-innovation, publicado em 1996 tido como o precursor dos estudos.

A inovação voltada para a sustentabilidade, ou ecoinovação, pode ser considerada como a introdução de produtos, processos produtivos, métodos de gestão ou negócios, novos ou significativamente melhorados para a organização e que traz benefícios econômicos, sociais e ambientais, comparados com alternativas pertinentes. Não se trata apenas de reduzir impactos negativos, mas de adicionar benefícios líquidos (FARIAS et al., 2016).

Pode-se assim entender que a eco inovação reveste-se de relevância para aquelas empresas que se preocupam em encontrar maneiras de diminuir impactos negativos que possam resultar dos processos produtivos das indústrias, servindo como uma ferramenta que propicia vantagem competitiva e gerando valor agregado aos produtos dada a imagem sustentável que possuem.

Ecoinovação relaciona-se intimamente com sustentabilidade cujo conceito foi difundido em estudo realizado pela Comissão Mundial sobre Meio Ambiente das Nações Unidas, conhecido como Relatório Brundtland (1987), que o conceitua como "desenvolvimento que satisfaz as necessidades atuais sem comprometer a habilidade das futuras gerações em satisfazer as suas necessidades" (SICHE et al., 2007).

As três dimensões que caracterizam sustentabilidade são a econômica, ambiental e social, também conhecidas como tripple bottom line interagem com o conceito de ecoinovações e fundem-se simbioticamente pois é impossível ser sustentável sem a presença ações ecoinovativas nos processos produtivos industriais.

De acordo com Kraemer (2003) para a existência de uma sociedade sustentável é necessário o 
desenvolvimento da sustentabilidade ambiental, social e política, construindo ajustes necessários durante esse processo com o objetivo de criar um equilíbrio entre eles. Para que não haja conflito entre esses valores e seja possível obter um resultado sustentável e economicamente significativo, é preciso que ocorra uma mudança de posicionamento e também exista uma orientação nos sistemas produtivos das organizações e da sociedade, com a produção e o consumo, minimizando os danos e impactos ambientais negativos causados ocasionalmente.

O conceito de desenvolvimento sustentável parte da premissa o meio ambiente é frágil e há necessidade de manter o equilíbrio dos ecossistemas. O debate em torno de se alcançar o desenvolvimento sustentável emergiu e foi direcionado para a finalidade de avaliar os desequilíbrios ambientais, sociais e econômicos, resultantes da relação 'crescimento econômico - meio ambiente (FARIAS et al., 2016).

Existe uma distinção entre os conceitos de preservação e conservação, os quais estão diretamente relacionados com as dimensões do desenvolvimento sustentável. Conservar implica com o uso racional de um recurso natural qualquer, sendo assim uma maneira de utilizar e ainda obter rendimentos economicamente viáveis e garantir a sua renovação e auto-sustentação.

A preservação ambiental relaciona-se com a ação de proteger, tornando-se mais restrita a utilização dos recursos, visando proteger contra destruição, danos ou degradações de uma área geográfica definida, espécies vegetais e animais ameaçados de extinção, ou bioma.

O cumprimento de requisitos legais faz com que a empresa seja menos propensa a causar danos ambientais, diminuindo os riscos de ser autuada e ter de pagar somas de vulto para recuperar os danos causados. No entanto, a questão é mais ampla pois não se trata mais de apenas evitar multas pecuniárias, mas agir com consciência ambiental, explorando de forma racional, não invasiva e preservacionista.

\section{Segmento Madeireiro}

O Brasil é o maior consumidor de madeira tropical no mundo, oriunda sobretudo dos estados do Pará, Mato Grosso e Rondônia. Os maiores consumidores internos são as regiões Sul e Sudeste. Só o estado de São Paulo consome mais do que o maior consumidor de madeira tropical na Europa, a França (SELING et al., 2002).

Especificamente, no Estado do Paraná, pesquisa realizada por Bittencourt et al. (2009) explicam que a atividade florestal e madeireira contribuiu efetivamente para o crescimento e desenvolvimento socioeconômico do Brasil e do Estado, fazendo parte de um dos ciclos econômicos mais importantes do Paraná.

Segundo dados da Associação Paranaense de Empresas de Base Florestal (APRE), o Estado do Paraná detém 967,0 mil ha plantados, sendo cerca de $70 \%$ (672,6 mil ha com pinus e $30 \%$ (294,1 mil ha) com eucalipto com expansão dos plantios florestais devendo-se notadamente ao eucalipto, que apresentou crescimento nos últimos anos na ordem de 9,2\%.

O Paraná lidera o ranking de maior detentor de área plantada com pinus do país e situa-se em sexto lugar na área plantada com eucalipto, totalizando área total plantada de 1.066 .479 ha, composta por $67 \%$ 
pinus (710.741 ha) e 33\% eucalipto (355.737 ha). A área florestal plantada no Paraná está concentrada na região Centro-Sul $(83,5 \%)$ e em menor proporção nas regiões Norte $(8,1 \%)$, Oeste $(4,3 \%)$, Noroeste $(2,9 \%)$, Centro-Oeste (1,0\%) e Litoral (0,2\%).

A demanda do setor madeireiro representa um retorno financeiro considerável, fazendo com que as empresas venham a experimentar constantes mudanças com propostas de novas gestões, uma vez que as empresas precisam se relacionar com outras empresas, com a sociedade e com o meio ambiente onde se localizam (REBOLLO, 2000).

\section{DISCUSSÃO TEÓRICA}

\section{Métodos e apresentação de dados}

A pesquisa classifica-se como etnográfica, com abordagem qualitativa e exploratória (WILLIS, 1977; WOODS, 1986; LÉVI-STRAUSS, 1988; GEERTZ, 1989; ERICKSON, 1984; MEHAN, 1992; MATTOS, 2011).

Utilizou-se, como instrumento de coleta de dados, entrevistas com roteiro pré-estruturado, cujas perguntas versaram sobre ações ecoinovativas implementadas nas empresas madeireiras. Aspectos ambientais, sociais e econômicos fizeram parte do escopo da análise. A população é composta por empresas do ramo madeireiro do Estado do Paraná e a amostra restringe-se às empresas localizadas geograficamente na Região Centro-Sul do território, totalizando 13 (treze) respondentes. Foram coletadas informações pessoais apenas para caracterizar o perfil dos respondentes, cujos dados foram tratados conjuntamente, conforme Termo de Consentimento Livre e Esclarecido (TCLE) e recomendações da Comissão Nacional de Ética em Pesquisa (CONEP).

Com relação ao perfil dos respondentes, observa-se que $61,5 \%$ (08) possuem terceiro grau completo, aproximadamente $31 \%(04)$ possuem terceiro grau incompleto e 01 respondente possui apenas 20 grau. No entanto, todos os respondentes atuam no setor financeiro e/ou administrativo das empresas consultadas.

Quando questionados sobre o grau de conhecimento sobre as inovações dentro do processo produtivo, $69 \%$ dos respondentes afirmaram possuir alto grau. Por outro lado, $41 \%$ aproximadamente responderam possuir conhecimento médio sobre os processos. Especificamente sobre o grau de conhecimento de ecoinovação, as respostas foram de $77 \%$ (10 respostas) para conhecimento médio e $23 \%$ afirmam não possuir conhecimento algum sobre o tema.

Com relação à questão: possui conhecimento se a empresa em que você trabalha já implantou alguma econovação? Os respondentes afirmaram que sim, a empresa implementou ações consideradas ecoinovativas no montante de 46\%, aproximadamente. No entanto, 3 respondentes afirmaram não haver ações desse tipo nas empresas e 4 respostas não possuírem conhecimento.

Dando sequência, caso a resposta da questão anterior fosse positiva, o questionamento foi se a empresa realizou analise de custo/benefício da implantação. Obteve-se três respostas afirmativas, ou seja, 50\% das empresas onde houve implantação de ações ecoinovativas, declararam relevante a análise.

Após, os respondentes foram questionados sobre qual o resultado quanto a efetividade das 
ecinovações em relação a benefícios econômicos, sociais e ambientais. Quatro respondentes afirmaram haver benefícios seja em virtude da melhoria das condições de trabalho, de diminuição da poluição do meio ambiente, da maior participação dos empregados nas decisões e consequente maior interação e motivação, seja por meio de ações de responsabilidade social.

Questionados sobre a relevância de ações que propiciam cuidados com o meio ambiente seja preservação e/ou recuperação todos os respondentes consideraram ser importante ou muito importante. No entanto, aproximadamente $23 \%$ dos respondentes, em entrevista, afirmaram às pesquisadoras que se preocupam mais com a "quantidade de veneno nas águas que contamina os rios e acaba com os peixes".

A empresa possui certificação de sistema de gestão ambiental pelo padrão ISO 14000 e/ou a certificação do Forest Stewarship Council (FSC) e/ou da Administração da Qualidade Ambiental Total (Total Quality Environmental Management -TQEM) 40\% dos respondentes afirmaram não terem conhecimento de tais certificações e normas. Isso demonstra uma dificuldade de comunicação interna, pois os empregados deveriam estar cientes não apenas da existência de tais normatizações bem como sobre seu teor, pois implicam necessariamente em ações coletivas e de processos.

\section{CONCLUSÕES}

A pesquisa teve por objetivo identificar os impactos causados pela implementação de ações ecoinovadoras dentro do processo produtivo de empresas do segmento madeireiro da Região Centro Sul do Paraná. Nesse sentido, apurou-se o conhecimento sobre ecoinovação, bem como a efetividade das ações implementadas pelas empresas pesquisadas.

Os resultados apontam para uma maior conscientização e cuidado do meio ambiente, por parte dos gestores, quando e na hipótese de alterações nos processos produtivos. Cuidado com o meio ambiente além da valorização do produto no mercado externo, propicia, preventivamente, a não exposição às multas pecuniárias por desrespeito às normas vigentes.

Diante dos resultados da pesquisa é possível inferir que há um aumento na conscientização dos empresários do segmento madeireiro sobre a relevância dos aspectos relacionados à inovação, bem como sociais e ambientais.

No entanto, existe um longo caminho a se percorrer seja na comunicação interna onde alguns respondentes não souberam explicar com exatidão sobre ações desenvolvidas pelas empresas em que trabalham denotando falhas, ou mesmo na discussão do que seja, de fato e de direto, ações ecoinovativas. Verifica-se ainda que embora existam legislações rígidas quanto às questões ambientais, há muito que evoluir seja na disseminação do conceito seja na implementação efetiva de ações que envolvam todos os atores das empresas do setor, em uníssono, em defesa de melhores condições de trabalho, remuneração digna e respeito ao meio ambiente.

\section{REFERÊNCIAS}

ARUNDEL, A.; KEMP, R.. Measuring eco-innovation. United Nations University: Maastricht Economic and social.
Research and training centre on Innovation and Technology, n.17, 2009. 
BARBIERI, J. C.; VASCONCELOS, I. F. G.; ANDREASSI, T.; VASCONCELOS, F. C.. Inovação e sustentabilidade: novos modelos e proposições. RAE-Revista de Administração de Empresas, v.50, n.2, 2010. DOI: http://dx.doi.org/10.1590/S0034-75902010000200002

BARBIERI, J. C.. Gestão ambiental empresarial: conceitos, modelos e instrumentos. São Paulo: Saraiva, 2011.

BITTENCOURT, L. P.; OLIVEIRA, G. B.. A indústria madeireira paranaense nos anos recentes. Revistas das Faculdades Santa Cruz, v.07, n.01, p.33-42, 2009.

ERICKSON, F.. What makes school ethnography 'ethnographic'?. Anthropology Educational Quarterly, v.15, p.51-66, 1984.

FARIAS, A. S. D.; MEDEIROS, H. R. D.; CÂNDIDO, G. A.. Contribuições de eco inovações para a gestão ambiental de atividades produtivas em um empreendimento da construção civil. Revista Adm. UFSM, Santa Maria, v.9, n.1, p.102-120, 2016.

FARIAS, A. S.; COSTA, D. S.; FREITAS, L. S.; CÂNDIDO, G. A.. Eco-innovation use of manufacturing in the process of red ceramic. RAI, v.9, n.3, p.154, 2012.

GEERTZ, C.. A interpretação das culturas. Rio de Janeiro: LTC, 1989.

KOELLER, P.; MIRANDA, P.; LUSTOSA, M. C.; PODCAMENI, M. G.. Ecoinovação: revisitando o conceito. 2020.

KNEIPP, J. M.; ROSA, L. A. B.; CICHUETI, R. S.; MADRUGA, L. R. R. G.; SCHUCH JÚNIOR, V. F.. Emergencia temática da inovação sustentável: uma análise da produção científica através da base web of science. Revista de Administração da UFSM, v.4, n.3, p.442-457, 2011.

KRAEMER, M. E. P.. Gestão dos Custos da Qualidade Ambiental. In: CONGRESSO BRASILEIRO DE CUSTOS, 9. Anais. São Paulo, 2003.

LAWSON, B.; SAMSON, D.. Developing innovation capability in organisations: a dynamic capabilities approach. International journal of innovation management, v.5, n.03, p.377-400, 2001.

LÉVI-STRAUSS, C.. Tristes trópicos. Barcelona: Paidós, 1988.

MATTOS, C. L. G.. A abordagem etnográfica na investigação científica. In: MATTOS, C. L. G.; CASTRO, P. A.. Etnografia e educação: conceitos e usos. Campina Grande: EDUEPB, 2011.

MAÇANEIRO, M. B.; CUNHA, S. K.. Eco-inovação: um Quadro de Referência para Pesquisas futuras. In: SIMPÓSIO DE GESTÃO DE INOVAÇÃO TECNOLÓGICA, 26. Anais. Anpad, 2010.

MAÇANEIRO, M. B.; CUNHA, S. K. Relações entre fatores contextuais internos às organizações e a adoção de estratégias proativas e reativas de ecoinovações. RAM-

Revista de Administração Mackenzie, v.16, n.3, p.20-50,

2015. DOI: https://doi.org/10.1590/1678-

69712015/administracao.v16n3p20-50

MEHAN, H.. Understanding inequality in schools: the contribution of interpretative studies. Sociology of Education, v.62, n.1, p.265-286, 1992.

OECD. Eco-Innovation in Industry: Enabling Green Growth. Paris: OECD Publishing, 2009.

PINSKY, V. C.; MORETTI, S. L. A.; KRUGLIANSKAS, I.; PLONSKI, G. A.. Inovação sustentável: uma perspectiva comparada da literatura internacional e nacional. Innovation and Management Review, v.12, n.3, p.226-250, 2015.

REBOLLO, M. G.. A contabilidade como instrumento de controle e proteção do meio ambiente. In: CONGRESSO BRASILEIRO DE CONTABILIDADE, 15. Anais. Goiânia: CFC, 2000.

RENNINGS, K.; ZWICK, T.. Employment impacts of cleaner production. ZEW Economic Studies, v.21, 2003.

SALES-CAMPOS, C.; ABREU, R. L. S.; VIANEZ, B. F.. Industrias madeireias de Manaus, Amazonas, Brasil. Acta Amazonica, v.30, n.2, p.319-331, 2000.

SCHIEDERIG, T.; TIETZE, F.; HERSTATT, C.. Green innovation in technology and innovation management: an exploratory literature review. ReD Management, v.42, n.2, p.180-192, 2012.

SELING, I.; KAISER, B.; SPATHELF, P.. Chancen der Vermarktung von zertifiziertemHolz auf dem inländischen Markt in Tropenwaldländern am Beispiel Brasilien. Forstarchiv, v.73, n.1, p.23-29, 2002.

SHARMA, S.; PABLO, A. L.; VREDENBURG, H.. Corporate environmental responsiveness strategies: the importance of issue interpretation and organizational context. The Journal of Applied Behavioral Science, v.35, n.1, p.87-108, 1999. DOI: http://dx.doi.org/10.1177/0021886399351008

SPAARGAREN, G.. Sustainable Consumption: a theoretical and environmental policy perspective. Society \& Natural Resources, v.16, p.687-701, 2003.

SPATHELF, P.; MATTOS, P. P.; BOTOSSO, P. C.. Certificação florestal no brasil uma ferramenta eficaz para a conservação das florestas naturais?. Floresta, v.34, n.3, 2004. DOI: http://dx.doi.org/10.5380/rf.v34i3.2423

UNEP. United Nations Environment Programme. The Business Case for Ecoinnovation Report. PNUMA, 2014.

WILLIS, P.. Learning to labor: working class kids get working class jobs. Farnborough: Saxon House, 1977.

WOODS, P.. Inside schools. Ethnography in educational resear. Routledge, 1986.

A CBPC - Companhia Brasileira de Produção Científica (CNPJ: 11.221.422/0001-03) detém os direitos materiais desta publicação. Os direitos referem-se à publicação do trabalho em qualquer parte do mundo, incluindo os direitos às renovações, expansões e disseminações da contribuição, bem como outros direitos subsidiários. Todos os trabalhos publicados eletronicamente poderão posteriormente ser publicados em coletâneas impressas sob coordenação da Sustenere Publishing, da Companhia Brasileira de Produção Científica e seus parceiros autorizados. Os (as) autores (as) preservam os direitos autorais, mas não têm permissão para a publicação da contribuição em outro meio, impresso ou digital, em português ou em tradução. 